\title{
Energy recovery linacs in high-energy and nuclear physics ts
}

\author{
Ilan Ben-Zvi ${ }^{a}, *$, Ya. Derbenev ${ }^{b}$, V.N. Litvinenko ${ }^{a}$, Lia Merminga ${ }^{\mathrm{b}}$ \\ a Brookhaven National Laboratory, MS 911B, Upton, NY 11973, USA \\ ${ }^{\mathrm{b}}$ Thomas Jefferson National Accelerator Facility, Newport News, VA 23606, USA
}

Available online 28 November 2005

\begin{abstract}
Energy recovery linacs (ERL) have significant potential uses in high energy physics and nuclear physics. We describe some of the potential applications which are under development by our laboratories in this area, and the technology issues that are associated with these applications.

The applications that we discuss are electron cooling of high-energy hadron beams and electron-nucleon colliders. The common issues for some of these applications are high currents of polarized electrons, high-charge and high-current electron beams and the associated issues of high-order modes. The advantages of ERLs for these applications are numerous and will be outlined in the text. It is worth noting that some of these advantages are the high brightness of the ERL beams and their relative immunity to beam-beam disturbances. (C) 2005 Published by Elsevier B.V.
\end{abstract}

PACS: 29.17. +w; 29.27.-a; 29.27. Hj; 29.90. +r

Keywords: Energy recovery linac; High average current; Superconducting RF; Collider

\section{Introduction}

Since the success of the Jefferson Laboratory series of FELs [1] in showing the great potential of the energy recovery linac (ERL), many applications have been proposed for ERLs in various science applications. In this paper, we concentrate on applications of the ERL for highenergy and nuclear physics.

The first application we discuss is electron cooling (EC) of hadron beams. The objective is to reduce the emittance and counteract emittance growth mechanisms inherent to stored hadron beams. This increases the luminosity and/or the luminosity lifetime of the hadron beam assumed to be used for collisions with another beam. In this application one brings the electron beam to interact with a stored hadron beam. Heat is transferred from the hadrons to the cooler electrons. The electron energy is recovered in the ERL accelerator and used to accelerate new bunches. The hadrons lose heat continu-

\footnotetext{
Work supported by the Office of Nuclear Physics, US Department of Energy.

*Corresponding author. Tel.: + 16313445143.

E-mail address: ilan@bnl.gov (I. Ben-Zvi).
}

ously and cool until some equilibrium is reached, typically with intra-beam scattering (IBS). The electron beam in this application is characterized by a relatively low energy (few $10 \mathrm{~s}$ of $\mathrm{MeV}$ ) but very high charge and very high current, and low emittance (considering the high charge).

The second application is the collision of high-current, high-charge electrons (often highly polarized) with hadron beams. The great advantage of ERLs in this application is the fact that the electron beam is discarded following energy recovery, and is thus much more immune to disruption due to collisions with the hadrons as compared to stored electron beams. The ERL beams for this application are characterized by high-charge, high-current and high-energy as well as polarization. ERL-based colliders thus offer a very high luminosity, and are in a position to help and unravel some of the crucial questions that remain open in nuclear physics:

What is the structure of the proton and neutron in terms of their quark and gluon constituents?

How do quarks and gluons evolve into hadrons?

What is the quark-gluon origin of nuclear binding?

How do the quarks and gluons manifest themselves in the properties of atomic nuclei? 
The two ERL designs that address these scientific issues are described below. The first is eRHIC [2,3] and the other is ELIC [4].

\section{Technology challenges}

The technology issues can be broken down to two areas. First, the generation of the necessary electron beams, then the acceleration of these beams, overcoming such issues as beam breakup and safe removal of HOM power from the superconducting RF (SRF) cavities.

The generation of beams for EC places the emphasis on high-charge, up to $20 \mathrm{nC}$, high-current, up to 0.5 amperes, strong magnetization, up to $5 \mathrm{~T} \mathrm{~mm}^{2}$ and low emittance, about $30-40 \mu \mathrm{m}$ (normalized $\mathrm{rms}$ emittance). One approach that is being followed at BNL [5] is the use of SRF laser photocathode gun, such as shown in Fig. 1. The high electric fields of the SRF cavity allow such an electron gun to generate the high-charge bunches with a good emittance.

The generation of polarized electron beams for nuclear physics is well known and used routinely at Jefferson Laboratory. The challenge of the ERL application comes from the need for both high-charge and high-current. The current density presently achieved from the surface of cesiated, strained gallium arsenide photocathodes are sufficiently high to be scaled to the required current (the requirements will be discussed in the collider sections of this paper). Such area scaling is transparent to cathode lifetime due to ion bombardment and conductivity issues. However, the integrated current is high. This requires a high laser power. It seems that adequate laser power can be provided either by lasers or, certainly, by high-power FELs. However, one issue that must be investigated is the stable acceleration and propagation of the current from the gun. The difficulty is that beam loss in the transport system leads to gas evolution from the struck surfaces. Since the gun must be operated at an ultra-high vacuum of better than $10^{-11}$ Torr, advances must be made in the production of beams with a reduced halo.

The acceleration of high-charge and high-averagecurrent beams in SRF structures is closely related to the problems associated with high-order modes (HOM). Dipole-type HOMs lead to the multi-bunch, multi-pass beam breakup (BBU) in ERLs. In addition, the large power generated by the HOMs must be safely removed from the cavities and dumped. Good damping of all modes is thus essential both for a high current threshold of the BBU and for the operation of the cryogenic SRF cavities. The HOM power is proportional to the product of the longitudinal loss factor $k_{1}$ by the average current and bunch charge. For a typical SRF cavity $k_{1}$ of $10 \mathrm{~V} / \mathrm{pC}$, a beam with $20 \mathrm{nC}$ bunch charge at $0.2 \mathrm{~A}$ average current will generate $40 \mathrm{~kW}$ of HOM power! This amount of power is higher than demonstrated so far. However, if the cavity loss factor can be reduced to $1 \mathrm{~V} / \mathrm{pC}$, the $\mathrm{HOM}$ power will be only $4 \mathrm{~kW}$, a value that can be handled by existing HOM dampers.

Fig. 2 shows a cavity designed specifically [6] for very high charge and average current SRF ERL service. The cavity is characterized by a low longitudinal loss factor of $1.1 \mathrm{~V} / \mathrm{pC}$, thanks to the combination of low frequency and very large apertures of $19 \mathrm{~cm}$ diameter. All HOM modes are damped to Qs of better than $10^{4}$ (most down to $10^{2}$ ) thanks to the large apertures, even larger beam pipe $(24 \mathrm{~cm}$ diameter) and beam-line ferrite damper. Simulations using the Jefferson Lab TDBBU and MATBBU codes show a $2 \mathrm{~A}$ threshold current for a small $50 \mathrm{MeV}$ ERL using such cavities. At the same time, the other performance measures of the cavity are excellent-a peak surface field to

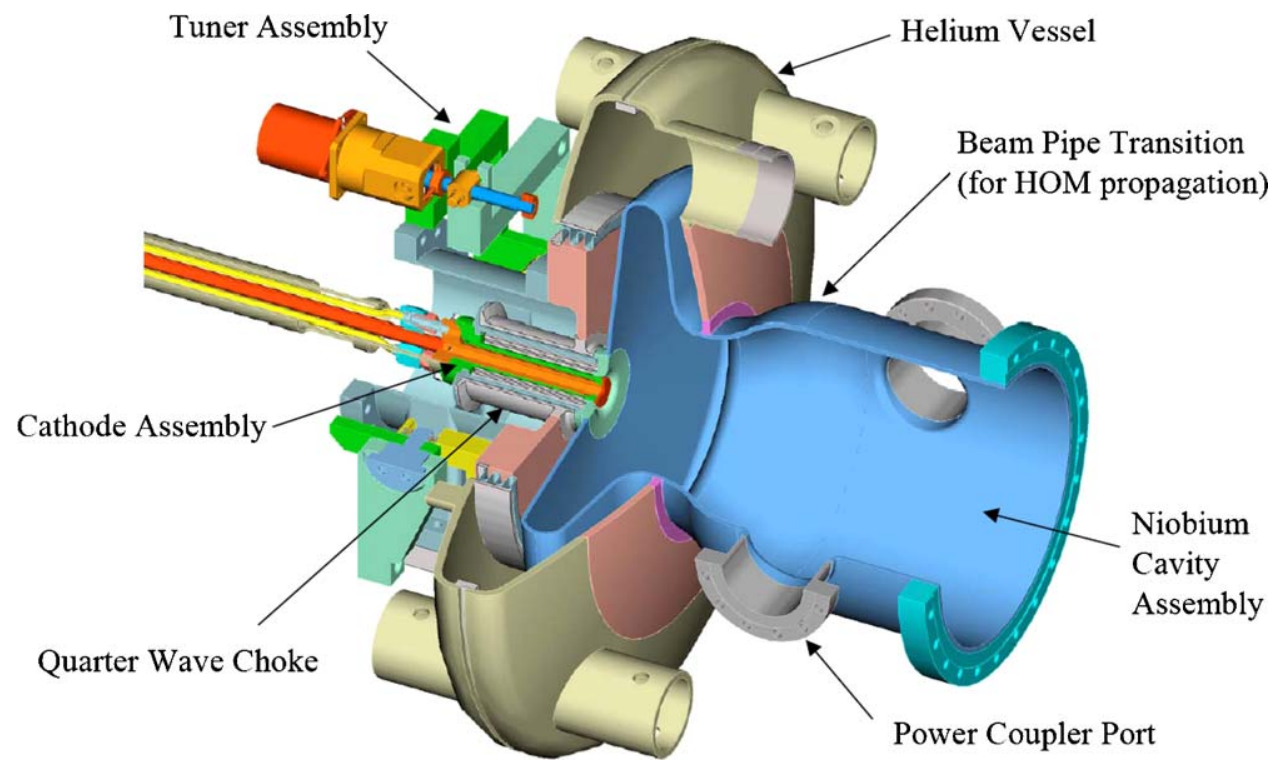

Fig. 1. Cutaway view of a $703.75 \mathrm{MHz}$ SRF laser-photocathode electron gun for high-charge, high-average current with a high brightness. 


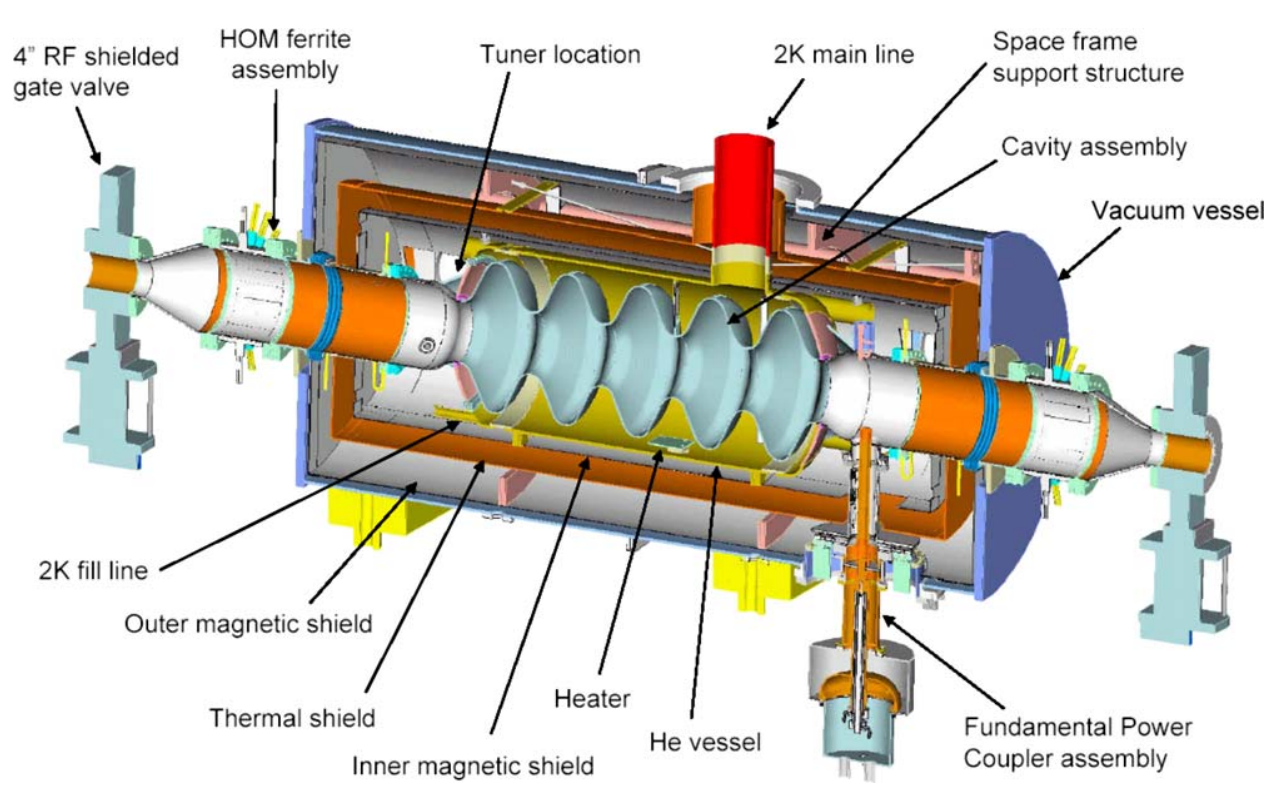

Fig. 2. Cutaway view of a $703.75 \mathrm{MHz}$ five-cell linac cryomodule. The low frequency and large apertures of this cavity as well as its special design couple all HOM power to ferrite dampers located on the beam pipes outside the cryomodule.

accelerating field ratio of 2 and very high stiffness (lowest mechanical mode at $100 \mathrm{~Hz}$ and Lorentz force detuning of $\left.1.2 \mathrm{~Hz} / \mathrm{MV} \mathrm{m}^{-2}\right)$.

\section{Electron Cooling}

Electron coolers have been around for about $40 \mathrm{yrs}$, but have changed very little until recently. Extant electron coolers can be characterized by the following: they operate at electron energies of a few tens to a few hundreds of $\mathrm{keV}$; they use highly magnetized DC thermionic electron guns; the electron beams are not bunched; the ions being cooled are stored but not collided with other beams. The recent deviation from this pattern is the FNAL recycler electron cooler [7], which is now under commissioning. This cooler uses an electron energy of $4.5 \mathrm{MeV}$ and non-magnetized electron beams. Cooling a higher-energy hadron beam calls for much higher electron beam energy. The Relativistic Heavy Ion Collider (RHIC) gold beam at $100 \mathrm{GeV} / \mathrm{A}$, requires an electron beam energy of $54 \mathrm{MeV}$. Since the cooling rate slows as a high power of relativistic energy factor $\gamma$ (about $\gamma^{7 / 2}$ ), it is clear that cooling at $\gamma \sim 100$ is a challenge.

Taking the RHIC electron cooler, which is at an R\&D phase, as an example of the highest energy electron cooler, [8] it is clear that the electron energy cannot be produced by an electrostatic accelerator. The use of an ERL suggests itself, thanks to the combination of high-current and good beam quality possible in such a machine. The R\&D work on cooling RHIC leads to the following electron beam parameters: energy of $54 \mathrm{MeV}$, bunch charge of $20 \mathrm{nC}$, bunch repetition frequency of $9.4 \mathrm{MHz}$, energy spread of the order of $10^{-4}$, emittance better than $40 \mu \mathrm{m} \mathrm{rms}$ normalized, magnetization of about $2-5 \mathrm{~T} \mathrm{~mm}^{2}$. A schematic drawing of the electron cooler is shown in Fig. 3. It consists of a gun (on the left of the lower ERL), merging optics, linear accelerator (along the bottom, including harmonic correction cavities), $\alpha$-shaped bunch stretcher, debunching cavity, solenoid, bunching cavity, $\alpha$-shaped bunch compressor and once again through the linac for energy recovery and then a beam dump.

The electron cooler at this energy is an expensive device with little margin for design errors. Thus a significant effort is ongoing at BNL, together with many collaborating institutes, to develop simulation codes that will predict the performance of the machine with some precision. Significant progress has been made in the development of the dynamics code BETACOOL [9] in collaboration with JINR, Dubna, the friction force from binary collisions code ParSEC [10] in collaboration with Tech-X, Colorado and others. In addition, a number of experiments carried out in the cooler ring Celsius and in the RHIC rings led to benchmarking of the various elements of the codes, from IBS to the friction force under various conditions, including the small Coulomb logarithm regime of RHIC.

The cooling of gold beams at RHIC leads to a luminosity increase as shown in Fig. 4, showing luminosity simulated with the code BETACOOL as a function of time with and without cooling. The non-cooled beam luminosity decays due to emittance growth and beam loss associated with intra-beam scattering. The cooled beam luminosity decays mainly due to beam loss at the collision point on account of the high luminosity. The integrated luminosity over a store period is about a factor of 10 larger than the non-cooled store.

\section{ERL-based polarized electron-hadron collider eRHIC}

A Twenty-Year Outlook DoE document on "Facilities for the Future Science" [11] includes a new electron- 


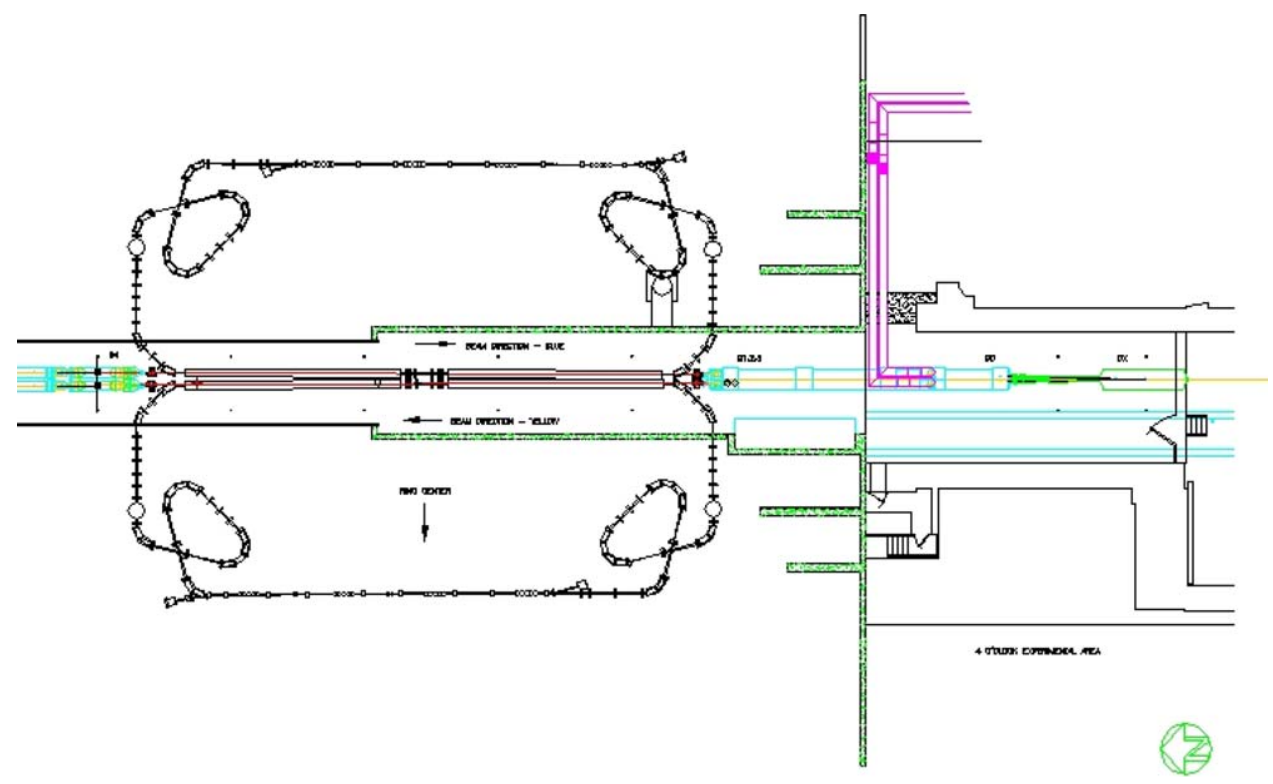

Fig. 3. Layout of the dual electron coolers planned for cooling the two RHIC ion storage rings. The solenoid magnets where the electrons exchange thermal energy with the ions are in a straight section of the ring, in proximity to one of the six interaction points.
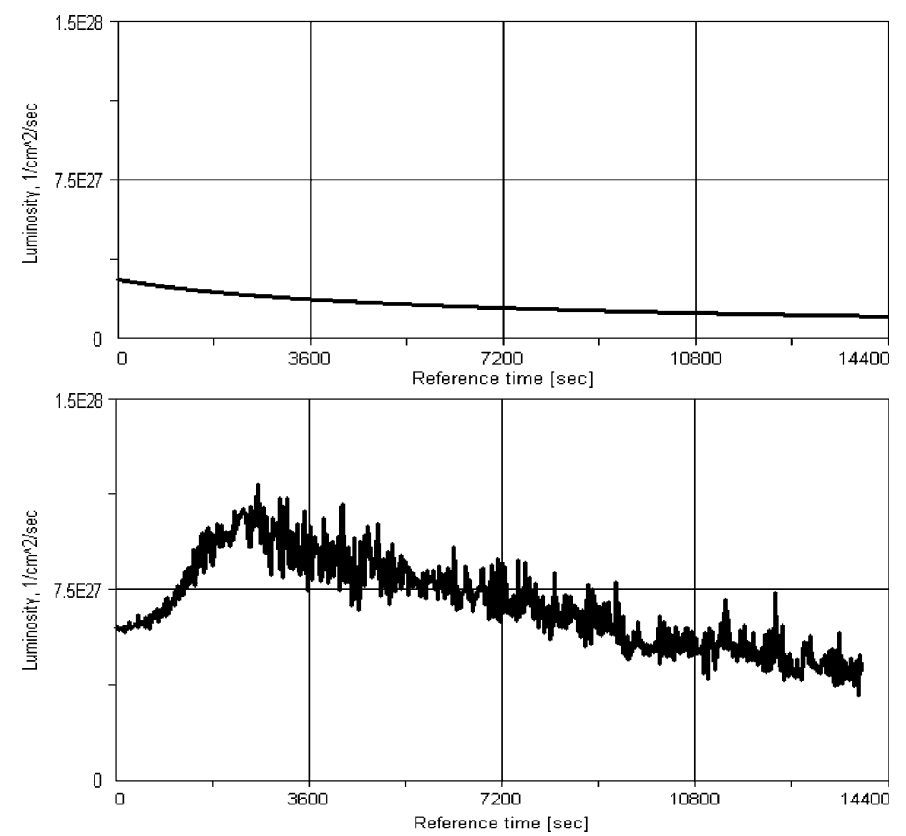

Fig. 4. Luminosity of gold-gold collisions at RHIC with and without electron cooling as a function of time.

hadron collider at BNL, based on the RHIC and a new electron accelerator. The main goal of the eRHIC is to explore the physics of quark-gluon matter at "low- $x$ ", and the physics of color glass condensate in electron-hadron collisions [12]. In response, the Collider-Accelerator Department at BNL in collaboration with Bates Laboratory at MIT issued the eRHIC ZDR $\left(0^{\text {th }}\right.$-order design report), which includes a 76-page-long linac-ring eRHIC design based on 10-20 GeV ERL [3]. The design is based on CW linac with high-current SRF five-cell cavities, which

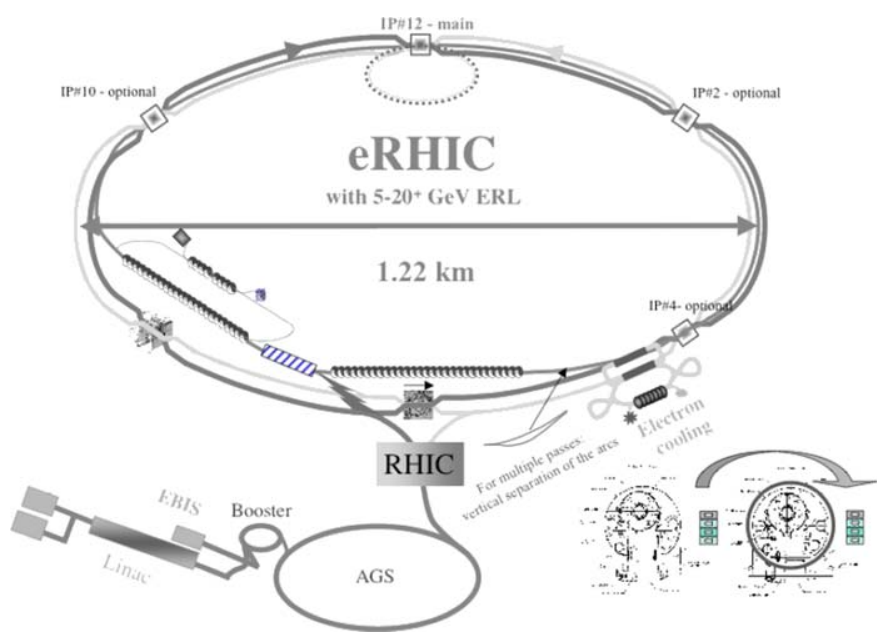

Fig. 5. Main layout of ERL-based eRHIC with up to four IPs and a possible $5 \mathrm{GeV}$ polarized positron storage ring. Polarized electrons are generated at a strained GaAs photocathode, accelerated in a multi-pass $\mathrm{ERL}$, used in one-to-four collisions with the hadrons counter-rotating in the RHIC blue ring, decelerated and finally dumped.

are described above and are under construction at BNL [5], and a polarized photo-injector using a dedicated $2 \mathrm{~kW}$ circularly polarized FEL [3]. The description of the eRHIC design based on the electron storage ring can be found in the ZDR [8].

Fig. 5 shows the main option for the high-energy eRHIC scenario with 2-20 GeV ERL whose return loops use the RHIC tunnel. This option provides for center of mass energies from $14 \mathrm{GeV}$ to above $140 \mathrm{GeV}$ in polarized electron-proton collisions. In the collisions of electron with gold ions, the energy in the center of mass (i.e. the electron-nucleon c.m. energy) will be from 20 to $90 \mathrm{GeV}$. 


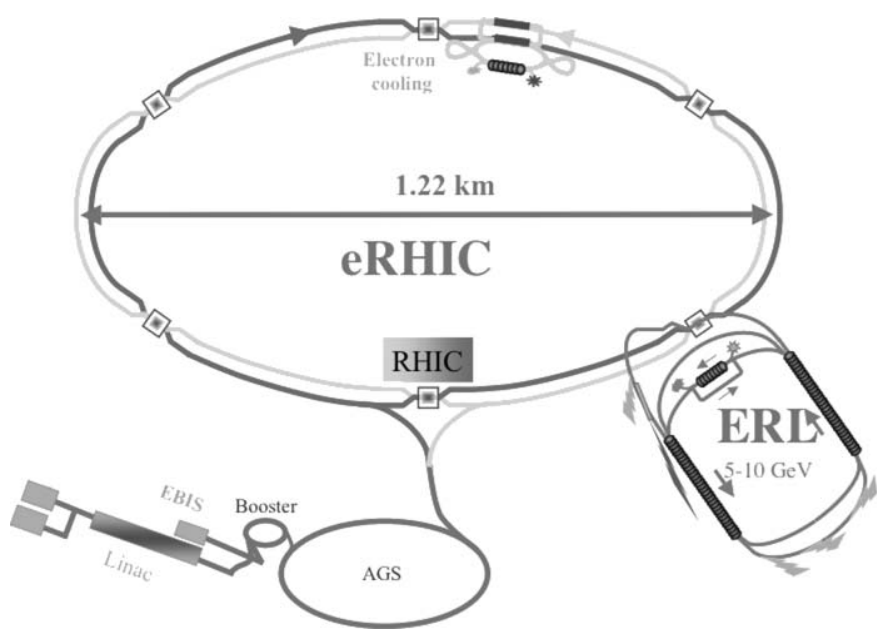

Fig. 6. eRHIC with a stand-alone $10 \mathrm{GeV}$ ERL.

Table 1

Parameters of ERL eRHIC

\begin{tabular}{ll}
\hline Ring circumference $(\mathrm{m})$ & 3834 \\
Number of bunches & 360 \\
Beam rep-rate $(\mathrm{MHz})$ & 28.15 \\
Hadrons: & \\
Beam energy (protons) $(\mathrm{GeV})$ & $26-250$ \\
Beam energy $\left(\mathrm{Au}_{197}^{79}\right)(\mathrm{GeV} /$ nucleon$)$ & $50-100$ \\
Protons per bunch $(\max )$ & $2.0 \times 10^{11}$ \\
Normalized $96 \%$ emittance $(\mu \mathrm{m})$ & 14.5 \\
rms bunch length $(\mathrm{m})$ & 0.2 \\
Beam energy $(\mathrm{GeV} / \mathrm{u})$ & $50-100$ \\
Ions per bunch $(\mathrm{max})$ & $2.0 \times 10^{9}$ \\
Normalized $96 \%$ emittance $(\mu \mathrm{m})$ & 6 \\
& \\
Electrons: & 28.15 \\
Beam rep-rate $(\mathrm{MHz})$ & $2-20$ \\
Beam energy $(\mathrm{GeV})$ & $3.9 \times 10^{3}-3.9 \times 10^{4}$ \\
Relativistic factor $(\gamma)$ & $5-50$ \\
rms normalized emittance $(\mu \mathrm{m})$ & $1.25-12.5$ \\
Beam emittance at $20 \mathrm{GeV}(\AA)$ & 30 \\
rms bunch length $(\mathrm{ps})$ & $0.1-1.0 \times 10^{11}$ \\
Electrons per bunch & $1.6-16$ \\
Charge per bunch $(\mathrm{nC})$ & 0.45 \\
Average e-beam current $(\mathrm{A})$ & \\
\hline
\end{tabular}

An option with a $2-10 \mathrm{GeV}$ stand-alone ERL, shown in Fig. 6, is also considered [3]. The main parameters of the ERL-based eRHIC are summarized in Table 1. The list of advantages provided by the ERL-based eRHIC compared with the ring-ring scenario [3] is rather long:

- usage of a fresh electron beam and absence of the memory in the e-beam practically waves the limitation on the tune shift in the IP, and allows increase in the intensity of proton/ion beam;

- increase in luminosity by 2-to-10 fold;

- larger $\beta^{*}$ for e-beam and simplified IP geometry;

- smaller e-beam emittance;

- smaller angular divergence in IP;
- smaller aperture for e-beam;

- no need for e-beam quads in the detector area;

- possibility to focus the e-beam after separating it from protons/ions;

- reduced number of coupled-bunch instability modes;

- spin transparency of the system at all energies;

- high $(80 \%+)$ degree of e-beam polarization at all energies;

- absence of "prohibited" energies for the e-beam;

- no need of preserving beam qualities (polarization, emittance, etc.) after the IP(s);

- simple geometry of the return pass;

- absence of spin resonances;

- possible multiple IPs;

- usage of the linac (ERL) geometry;

- easy adjustment of the e-beam repetition rate to the beam repetition rate in the $\mathrm{RHIC}$, which significantly depends on the ion energy (equivalent change in circumference is $\sim 30 \mathrm{~m}$ );

- easy future e-beam energy upgrades;

- possibility of using multiple energy collisions;

- possibility of $\gamma$-hadron collider with ERL-based Compton source of $\gamma$-rays.

In the ERL-based eRHIC we plan to collide two round beams of equal size to maximize the luminosity. The main distinctive feature here is that the attainable luminosity is defined in practice by the energy and intensity of the proton or ion beam in RHIC:

$L=f_{\mathrm{c}} \xi_{\mathrm{h}} \frac{\gamma_{\mathrm{h}}}{\beta_{\mathrm{h}}^{*}} \frac{Z N_{\mathrm{h}}}{r_{\mathrm{h}}}$,

i.e., by the intensity $N_{\mathrm{h}}$ (number of hadrons per bunch), repetition rate $f_{\mathrm{c}}$, the energy of the ion or proton beam, $\gamma_{\mathrm{h}}=E_{\mathrm{h}} / M c^{2}$, its charge $q=Z e$, its classical radius $r_{\mathrm{h}}=$ $Z^{2} e^{2} / M c^{2}$ and the allowable beam-beam tune shift $\xi_{\mathrm{h}}$ in the eRHIC IP(s). The linac-ring eRHIC's luminosity is independent of the electron beam's energy, and linearly proportional to the energy of the proton or ion beam. This means that the same center of mass energy, (given that there is no preferred energy ratio), can be reached using higher energy protons (ions) and lower energy electrons; hence, the high luminosity in contrast with the ring-ring scenario [6], where the electrons beam-beam tune shift limits the attainable luminosity. Details on the eRHIC IP design can be found in Ref. [13].

For the linac-ring collider, the beam-beam effect on the electron beam is better described not by a tune shift but by a disruption parameter, i.e. an additional betatron phase advance caused by the ion beam during the interaction. As seen in Fig. 7, the electron beam can survive very violent collisions with hadrons. The real limitation of the ERL based eRHIC may be limited by kick instability of the proton beam [14]. The proton beams in RHIC are rather long $(\sim 1 \mathrm{nsec})$ and we plan to develop a transverse feedback system to suppress the lowest modes of this instability. 

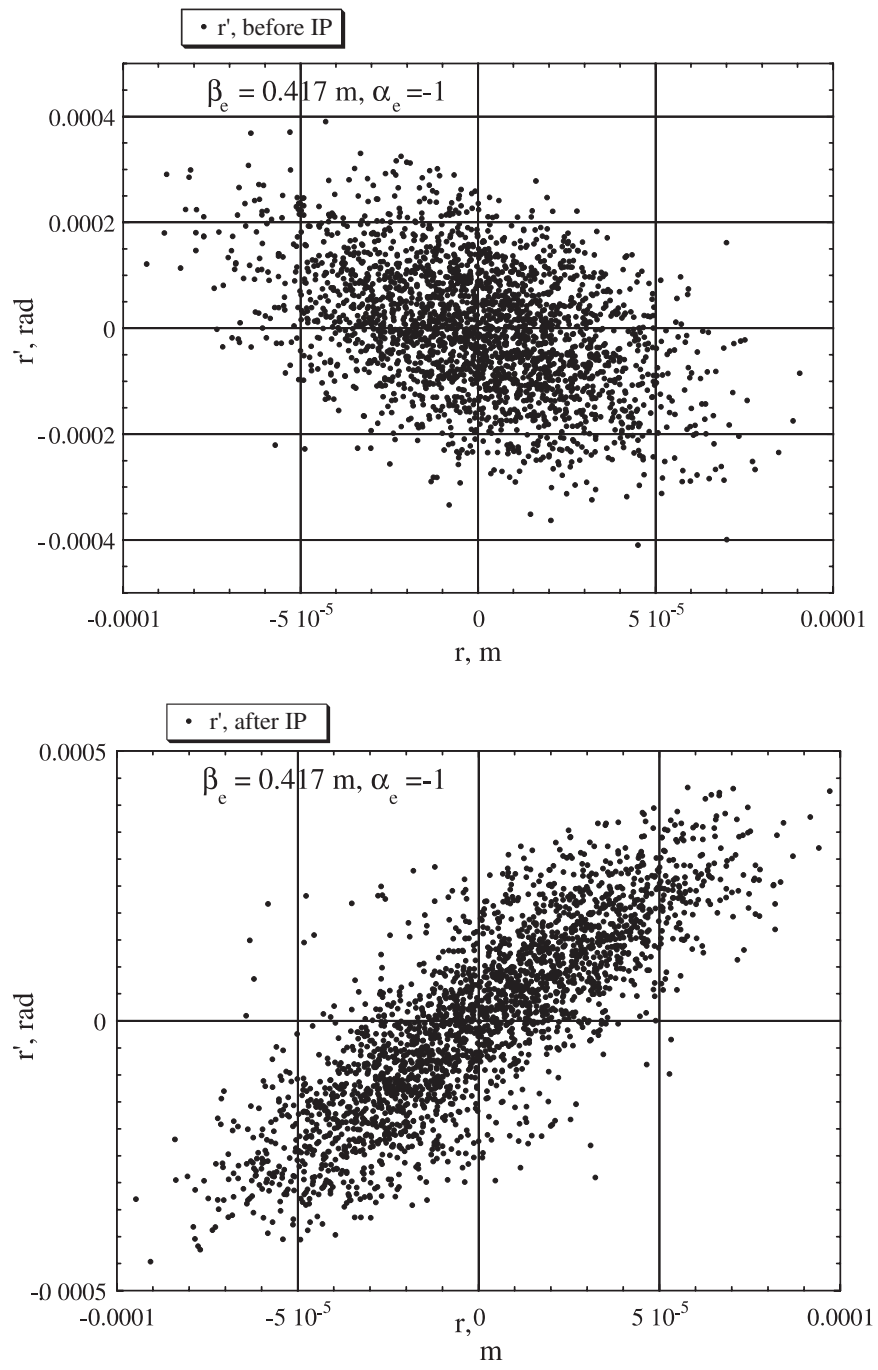

Fig. 7. Round $10 \mathrm{GeV}$ electron beam from ERL with initial transverse rms emittance of $3 \mathrm{~nm}$ rad passes through the IP with the disruption parameter 3.61 (tune shift $\xi_{\mathrm{xe}}=0.6$ ). The emittance growth is only $11 \%$. The top figure shows the phase-space distribution before the IP, and the bottom figure after the IP (ring/ERL case: $N_{\mathrm{Au}}=2 \times 10^{9} ; \mathrm{rms}$ $\left.\varepsilon_{i}=5 \mathrm{~nm} \mathrm{rad} ; \beta_{i}^{*}=0.25 \mathrm{~m} ; \sigma_{\mathrm{zi}}=0.20 \mathrm{~m} ; \varepsilon_{\mathrm{e}}=3 \mathrm{~nm} \mathrm{rad}\right)$, on the same scale.

The eRHIC will use GaAs photocathode driven by nearIR FEL for generation of a longitudinally polarized beam with a high degree $(>80 \%)$ of polarization [15]. We illustrate the spin manipulation in the ERL using a second layout of eRHIC shown in Fig. 8. Electrons are generated in a photo-injector with longitudinal polarization exceeding $80 \%$ and the energy of $E_{i}=\gamma_{i} m c^{2}$. Helicity is controlled by the choice of helicity of the photons, and can be switched from positive to negative. The electron beam is turned for the angle $\Delta \varphi_{i}=\pi / 12$ and is injected into the first stage of the ERL. The first stage of the ERL has one linac. Electron beam passes twice through it gaining $\Delta E_{1}=\Delta \gamma_{1} m c^{2}$ at each pass and makes one $360^{\circ}$ turn before reaching a transfer energy $E_{1}=\gamma_{1} m c^{2}$ into the second section of the ERL. The e-beam makes a $\Delta \varphi_{\mathrm{t}}=\pi / 2$ turn before reaching the first main acceleration section.

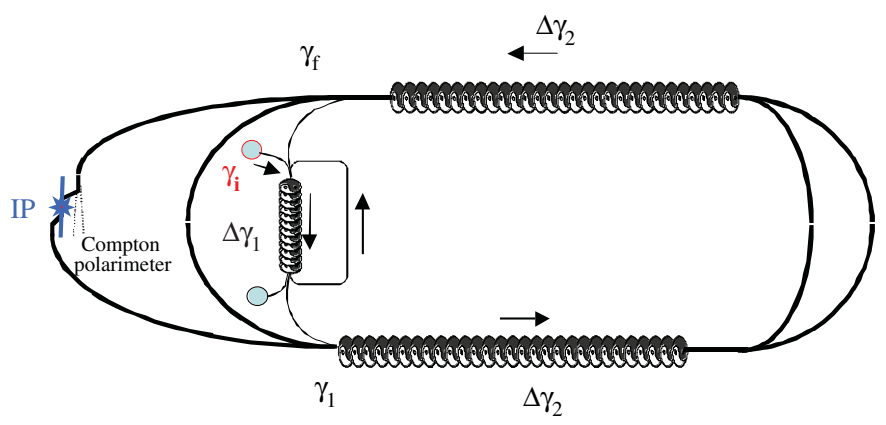

Fig. 8. Polarization transparency in the stand-alone ERL for eRHIC.

Table 2

Luminosities for $\mathrm{e}-\mathrm{p}$ collisions for various energies in the ERL-based eRHIC -360 bunches with $2 \times 10^{11}$ protons per bunch

\begin{tabular}{lllll}
\hline $\begin{array}{l}\text { Luminosity in } \\
10^{33} \mathrm{~cm}^{-2} \mathrm{~s}^{-1}\end{array}$ & Protons & & & \\
\cline { 2 - 5 } & $26 \mathrm{GeV}$ & $50 \mathrm{GeV}$ & $100 \mathrm{GeV}$ & $250 \mathrm{GeV}$ \\
\hline $\begin{array}{l}\text { Parallel mode with RHIC } \\
\text { collider }\end{array}$ & 0.285 & 0.548 & 1.097 & 2.74 \\
$\begin{array}{l}\text { Dedicated eRHIC mode } \\
\text { yed }\end{array}$ & 0.978 & 1.88 & 3.76 & 9.40 \\
\hline
\end{tabular}

The electron beam passes twice through each of the main accelerator sections $\Delta E_{2}=\Delta \gamma_{2} m c^{2}$ at each pass, and makes three $180^{\circ}$ turns before reaching the final energy $E_{\mathrm{f}}=\gamma_{\mathrm{f}} m c^{2}$. The e-beam makes one last $\Delta \varphi_{\mathrm{t}}=\pi / 2$ turn before reaching its goal in the IP. The spin rotation in ERL-based eRHIC is very simple - spin rotates for an angle of $\Delta \varphi_{\mathrm{s}}=\Delta \varphi \cdot \gamma \cdot a$ around the vertical axis, where $\Delta \varphi$ is the angle of rotation in a bend, $\gamma$ is the relativistic factor and $a=g / 2-1=1.1596521884 \times 10^{-3}$ is the anomalous magnetic moment of the electron. Changing $\Delta \gamma_{1}$ and $\Delta \gamma_{2}$ (within a few percentage range), while keeping the final energy, $\gamma_{\mathrm{f}}$, constant, one can rotate the spin at any desirable angle, i.e. achieve full spin transparency at any energy. A similar technique will be used for the main option of the ERL-based eRHIC (see Fig. 5).

The luminosities for $\mathrm{e}-\mathrm{p}$ collisions for various energies in the ERL-based eRHIC with 360 bunches and $2 \times 10^{11}$ polarized protons per bunch colliding with polarized electrons are shown in Table 2. The ERL-based eRHIC will satisfy all requirements set by the nuclear physics and spin program at RHIC and, in addition, will open a number of new opportunities. It will exploit the developing technology of high current, high brightness electron beam to realize the full potential of the polarized electron-positron collider in Collider-Accelerator Department at BNL. In addition, the quality of the electron beam in the ERL makes it a natural fourth-generation source of spontaneous and coherent radiation. These features of the ERL-based eRHIC are published elsewhere [16]. 


\section{ELIC - an electron-light ion collider based at CEBAF}

An ERL-based electron-light ion collider of c.m. energy from 20 to $65 \mathrm{GeV}$ and luminosity from $10^{33}$ to $10^{35} \mathrm{~cm}^{-2} \mathrm{~s}^{-1}$ with both beams polarized is envisioned as a future upgrade to CEBAF. The ELIC facility would produce a variety of polarized light ion species: $p, d,{ }^{3} \mathrm{He}$ and $\mathrm{Li}$ and unpolarized light to medium ion species. The highest bunch repetition rate (up to CEBAF's RF frequency of $1.5 \mathrm{GHz}$ ) is envisioned for maximum attainable luminosity. Longitudinally and transversally spin-polarized light ion beams in the ring at all energies, with the flexibility of switching from longitudinal to transverse spin in the detectors, as well as fast flipping of the spin are of critical importance to the science and are envisioned features of ELIC.

The parameters for ELIC at various c.m. energies are shown in Table 3. The concept of luminosity of ELIC has been established based on high energy EC, beam-beam interaction between electron beam from the linac and the stored ion beam, high bunch collision frequency, short ion bunches, very strong focus at the IP and the use of crab-crossing $[4,17,18]$.

To realize the required electron beam for ELIC, shown schematically in Fig. 9, it is proposed to develop CEBAF to a single recirculation $7 \mathrm{GeV}$ ERL with 3-30 $\mathrm{mA}$ polarized electron current from a photo-injector. This upgrade is deemed compatible with the CEBAF upgrade for a $24 \mathrm{GeV}$ fixed target program [19]. 2.4 A polarized electron beam colliding with ion beams is required to reach the full luminosity of ELIC. It is generally anticipated that the next generation electron guns will be able to produce up to $100 \mathrm{~mA}$ of polarized electrons, a 300 -fold increase of the present record of $300 \mu \mathrm{A}$. To get around this constraint, it was proposed to use a circulator-collider ring in the ELIC design. The electrons would go 100 turns in the circulator ring before being energy-recovered. Recently, a scheme of harmonic injection/extraction has been proposed to switch electron bunches between the ERL and the circulator ring. The scheme is described in Ref. [4] and is based on RF separator cavities which are presently in use at CEBAF. The proposal is similar to the one proposed for the
International Linear Collider Damping Ring, but requires much higher frequencies.

To attain the required ion beams, it is proposed to build an ion facility, a major component of which is a $150 \mathrm{GeV}$, 1 A collider ring with up to four interaction regions (IRs). The booster rings, electron circulator-collider ring and the ion collider ring are designed as a Fig. 8. Such configuration eliminates the issue of spin maintenance at acceleration and allows one to easily arrange the desired spin orientation and flipping for all ion species at all energies. To suppress ion beam blowup due to IBS and its impact on the luminosity, EC is introduced. An ERL-based highenergy EC concept similar to the one pursued at BNL, and described above, is invoked for ELIC. A variation of the ELIC cooling scheme is the use of a circulator-cooler ring as a way to reduce the necessary electron current in the $75 \mathrm{MeV}$ ERL. Other challenge of high-energy EC is the design of the electron beam transport system compatible with efficient acceleration and beam alignment. As an alternative to the classical solenoid transport-based EC, we also explored cooling with electron beam focused along the cooling section by quadrupoles but delivered from a nonmagnetized electron gun. The principal feature of such a scheme is that the electrons oscillating in the focusing field do interact with ions of the beam under cooling in a way

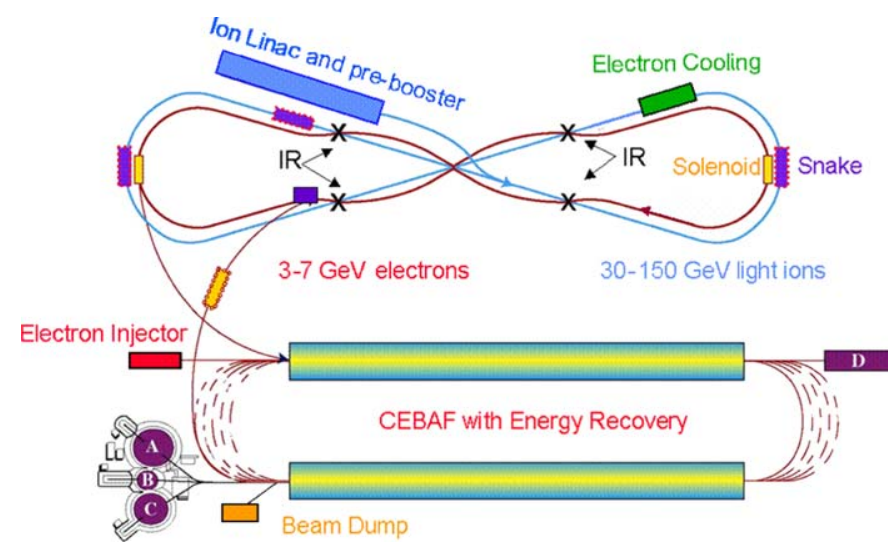

Fig. 9. Schematic layout of ELIC.

Table 3

Basic parameters for ELIC

\begin{tabular}{llll}
\hline Parameter & Unit & Value & Value \\
\hline Beam energy & $\mathrm{GeV}$ & $150 / 7$ & $100 / 5$ \\
Bunch collision rate & $\mathrm{GHz}$ & 1.5 & $30 / 3$ \\
No. of particles/bunch & $10^{10}$ & $0.4 / 1.0$ & $0.4 / 1.1$ \\
Beam current & $\mathrm{A}$ & $1 / 2.4$ & $1 / 2.7$ \\
Energy spread, rms & $10^{-4}$ & 3 & $0.12 / 1.7$ \\
Bunch length, rms & $\mathrm{mm}$ & 5 & $0.3 / 4.1$ \\
Beta-star & $\mathrm{mm}$ & 5 & $0.7 / 70$ \\
Horizontal emit. (norm.) & $\mu \mathrm{m}$ & $1 / 100$ & $0.06 / 6$ \\
Vertical emit. (norm.) & $\mu \mathrm{m}$ & $0.04 / 4$ & $0.01 / 0.073$ \\
Beam-beam tune shift (vertical) per IP & & $0.01 / 0.086$ & 0.03 \\
Space charge tune shift in p-beam & $\mathrm{cm}{ }^{-2} \mathrm{~s}^{-1}$ & 0.015 & 5.6 \\
Luminosity per IP* (10 & $\mathrm{hrs}$ ) & 7.7 & $0.2 / 43$ \\
Luminosity lifetime & & 24 & $0.2 / 43$ \\
\end{tabular}


similar to that of high-temperature electrons experiencing cyclotron motion in a solenoid. The cooling efficiency can be improved and optimized by scanning the electron beam around the ion beam area. The advantages of this concept are: (1) absence of long, heavy superconducting solenoid in the cooling section; (2) SRF gun can be employed; (3) the beams become easily accessible for diagnostics and alignment in the cooling section; and (4) heavy ion recombination practically disappears. EC is anticipated to provide low emittance and simultaneously very short ion bunches. The short bunches have two critical advantages: (1) a super-strong beam focusing at the collision points and (2) crab-crossing colliding beams.

To eliminate the parasitic beam-beam interaction, the colliding beams should intersect at some angle. To avoid luminosity loss, bunches then should be turned by half of the angle, thus becoming parallel to each other. Bunch tilt is produced by crab resonators. In the ELIC design, the $\mathrm{crab}$ resonators are installed before and after the outer final focus magnets, viz., they are centered at the outer focus points of an experimental area with two interaction points. Then, the colliding bunches do not rotate, while the crab tilt becomes compensated after the second resonator. Based on estimates, a dipole magnetic field resonator with an effective $1.5 \mathrm{GHz}$ SRF voltage of about $80 \mathrm{MV}$ is sufficient to create a $50 \mathrm{mrad}$ bunch tilt for a $150 \mathrm{GeV}$ proton beam and, yet, fit in the free space near the final focus magnets.

To implement the ultra-tight focusing of ions in the ELIC IR, it is beneficial to use a focusing triplet (DFD or FDF) which provides a net focal length of about $4.5 \mathrm{~m}$ at the collision energy of $150 \mathrm{GeV}$. This triplet uses two types of quadrupoles: $1 \mathrm{~m}$ long defocusing one and $2 \mathrm{~m}$ long focusing one, with a $3 \mathrm{~cm}$ transverse aperture radius and $7.5 \mathrm{~T}$ peak field. The quadrupole parameter defines a maximum field gradient of $250 \mathrm{~T} / \mathrm{m}$. The final focus lattice can be configured either symmetrically (DFDODFD) or anti-symmetrically (FDFODFD). The advantage of the anti-symmetric configuration is its lower sensitivity to ground motion, magnet power supply fluctuation, etc.

To optimize the luminosity for asymmetric emittance, our design assumes $\beta_{x}{ }^{*}=2.5 \mathrm{~cm}$ and $\beta_{y}{ }^{*}=0.5 \mathrm{~cm}$, with $\beta_{\max }=5200 \mathrm{~m}$.

The ELIC IR will consist of two final focusing points for two detectors separated by about a $60 \mathrm{~m}$ distance. The IR region will then be matched through another lattice with arcs where the $\beta$ function equals $12 \mathrm{~m}$ or less.

The IR for $7 \mathrm{GeV}$ electrons with $0.1 \mathrm{rad}$ vertical crossing angle is designed to interleave transversely with the focusing system for ions. Here, the optics is based on two doublets separated by a 9-m-long drift to accommodate quads of the ion part. Similar to the IR for ions, it assumes $\beta_{x}{ }^{*}=2.5 \mathrm{~cm}$ and $\beta_{y}{ }^{*}=0.5 \mathrm{~cm}$ with $\beta_{\max }=2600 \mathrm{~m} \mathrm{[20].}$

\section{Conclusions}

Energy recovery linacs provide a unique technology for high energy electron cooling and advanced concepts of electron-ion colliders required to answer fundamental questions in nuclear and particle physics. The advantages of these ERL-based devices are numerous, but the challenges are also respectable. Vigorous R\&D pursued at BNL, JLab and elsewhere, on the generation and stable and efficient acceleration of the high current beams, will realize the promise of ERLs and open a new era in nuclear physics research.

\section{References}

[1] G. Neil, these proceedings.

[2] I. Ben-Zvi, J. Kewisch, J. Murphy, S. Peggs, Accelerator physics issues in eRHIC, Nucl. Instr. and Meth. Phys. Res. A 463 (94) (2001) $\mathrm{C}-\mathrm{A} / \mathrm{AP} / 14$.

[3] D. Anderson, I. Ben-Zvi, R. Calaga, X. Chang, M. Farkhondeh, A. Fedotov, J. Kewisch, V. Litvinenko, W. Mackay, C. Montag, T. Roser, V. Yakimenko, http://www.agsrhichome.bnl.gov/eRHIC/ eRHIC_ZDR/AppendixA.pdf.

[4] Ya. Derbenev, et al., Proceedings of Particle Accelerator Conference, 15-20 May 2005, Knoxville, TN.

[5] I. Ben-Zvi, et al., Ampere average current photoinjector and energy recovery linac, Proceedings of 2004 International FEL Conference, 29 August-3 September 2004, Trieste, Italy, , http://accelconf.web.cern. ch/accelconf/f04/papers/THBOC04/THBOC04.PDF.

[6] R. Calaga, I. Ben-Zvi, Y. Zhao, J. Sekutowicz, High current superconducting cavities at RHIC, Proceedings EPAC'04, 5-9 July 2004, Lucerne, Switzerland, http://accelconf.web.cern.ch/AccelConf/ e04/PAPERS/TUPKF078.PDF.

[7] J.R. Leibfritz, et al., Status of the Fermilab electron cooling project, Proceedings EPAC'04, 5-9 July 2004, Lucerne, Switzerland, http:// accelconf.web.cern.ch/accelconf/e04/PAPERS/TUPLT151.PDF.

[8] Zero design report on electron cooling of RHIC, http://www. agsrhichome.bnl.gov/eCool/.

[9] The BETACOOL program, http://lepta.jinr.ru.

[10] D.L. Bruhwiler, et al., Direct simulation of friction forces for heavy ions interacting with a warm magnetized electron distribution, in: Proceedings of 33rd ICFA Advanced Beam Dynamics Workshop, 2004.

[11] http://www.er.doe.gov/Sub/Facilities_for_future/facilities_future.htm.

[12] T. Hallman, et al., RHIC II/eRHIC white paper submitted to the NSAC sub-committee on future facilities, 2003, http://www.bnl.gov/ henp/docs/NSAC_RHICII-eRHIC_2-15-03.pdf.

[13] C. Montag, et al., Beam-beam simulations for double-gaussian beams, Proceedings of Particle Accelerator Conference, 15-20 May 2005, Knoxville, TN.

[14] R. Li, B.C. Yunn, V. Lebedev, J.J. Bisognano, Proceedings of PAC 2001 (2001) p. 2014.;

E.A. Perevedentsev, A.A. Valishev, Phys. Rev. ST-AB 4 (2001) 024403; R. Li, B.C. Yunn, ICFA Beam Dynamics Newsletter No. 30, April 2003, p. 69

[15] P. Hartman, et al., Polarized electron linac sources, in: Proceedings of the Second eRHIC Workshop, 6-8 April 2000, Yale, CN, p. 120.; T. Zwart, et al., Polarized electron at Bates: source to storage ring, Proceedings of Second EPIC Workshop, 14-15 September 2000, Cambridge, MA, p. 343.

[16] V.N. Litvinenko, I. Ben-Zvi, Proceedings of FEL'2004 Conference, 30 August-3 September 2004, Trieste, Italy, pp. 594-597, http:// accelconf.web.cern.ch/AccelConf/f04/.

[17] L. Merminga, Ya. Derbenev, ELIC: a high luminosity and efficient spin manipulation electron-light ion collider at CEBAF, Proceedings of CIPANP Conference, 2003.

[18] Ya. Derbenev, et al., Proceedings of EPAC'04, 5-9 July 2004, Lucerne, Switzerland.

[19] Jefferson Lab Institutional Plan, FY 2004-2008, October 2004.

[20] C. Montag, et al., Proceedings of Particle Accelerator Conference, 15-20 May 2005, Knoxville, TN. 